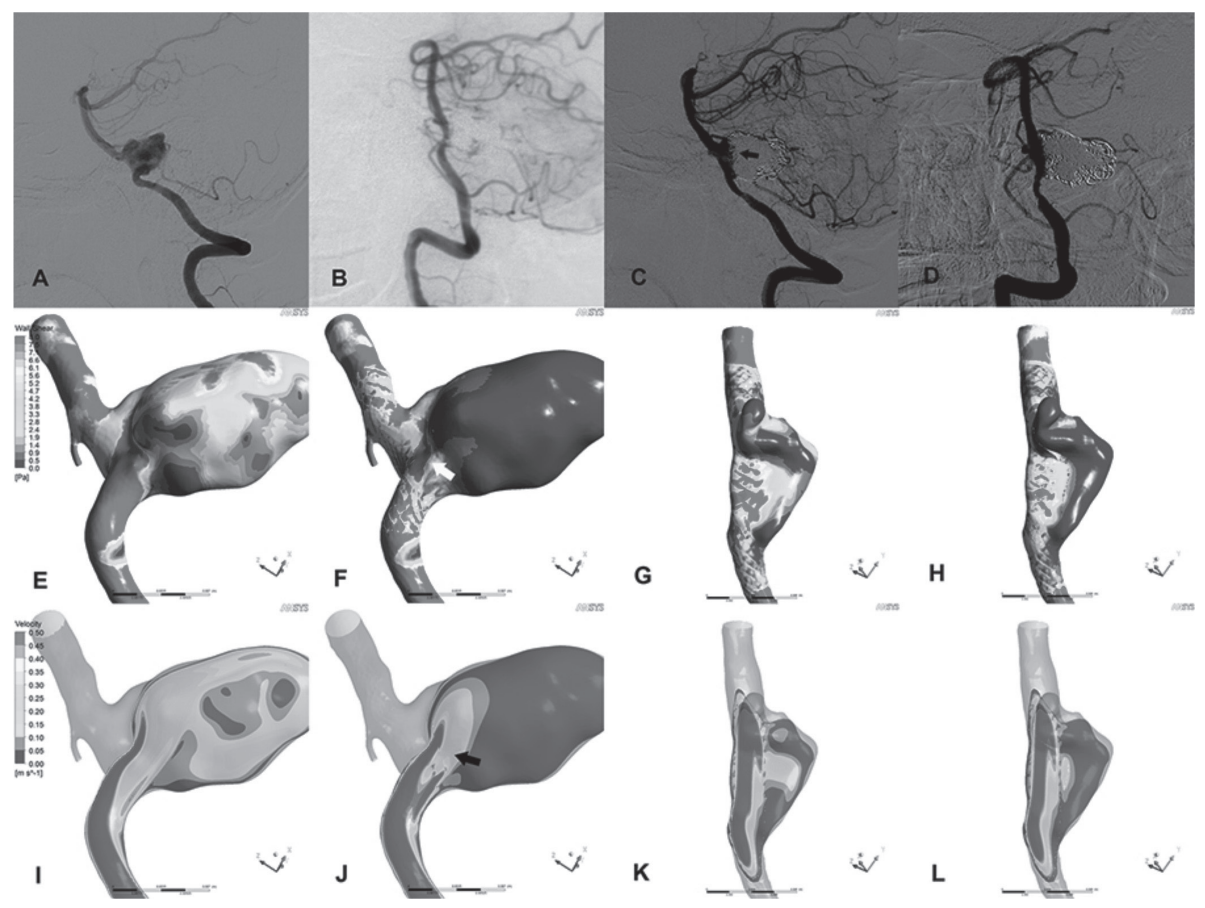

Abstract 0-017 Figure 2

Materials and methods Between September 2009 and November 2013, all consecutive patients presenting with recurrent VADAs after SACE in our institutions were enrolled in the study. Recurrence was defined as recanalization and regrowth. We assessed the hemodynamic alterations in WSS and velocity by CFD simulation after initial SACE and subsequently after retreatment of aneurysms that recurred.

Results In the study period, 112 consecutive patients with intracranial veterbrobasilar dissecting aneurysms (VBDAs) underwent endovascular treatment. Seventy-two of them were treated with SACE. Angiographic follow-up was available in 59 patients (81.9\%). Recurrence was present in 10 patients $(16.9 \%)$ and 6 needed retreatment. Finally, 5 patients with VADAs were included (1 was excluded because of inadequate 3D imaging). After initial treatment, three cases showed recanalization and 2 cases showed regrowth. In the 2 regrew cases, the 2 original aneurysms maintained complete occlusion, however de-novo aneurysm regrowth was confirmed near the previous site. Compared with 3 recanalised aneurysms, the completely occluded aneurysms showed high mean reductions in velocity and WSS after initial treatment $(77.6 \%$ versus $57.7 \%$ in velocity, $74.2 \%$ versus $52.4 \%$ in WSS), however, remaining high WSS at region near the previous lesion where the new aneurysm originated. After the second retreatment, there was no recurrence in all cases. Compared with the 3 aneurysms that recanalised, the 4 aneurysms that maintained complete occlusion showed higher reductions in velocity (62.9\%) and WSS (71.1\%).

Conclusion Hemodynamics by CFD may be useful in predicting recurrence of VADAs after SACE.

Disclosures J. Liu: None. L. Jing: None. Y. Zhang: None. Y. Song: None. Y. Wang: None. C. Li: None. Y. Wang: None. S. Mu: None. N. Paliwal: 1; C; National Institutes of Health (R01 NS091075). H. Meng: 1; C; National Institutes of Health (R01 NS091075). I. Linfante: None. X. Yang: 1; C;
National Natural Science Foundation of China (Grant No. 81301003, 81171079, 81371315, 81471167 and 81220108007), Special Research Project for Capital Health Development (Grant No. 2014-1-1071).

\section{0-018 SINGLE INSTITUTION EXPERIENCE WITH TWO FLOW DIVERTERS: A HEAD TO HEAD COMPARISON}

A Paul, J Dalfino, J Yamamoto, A Boulos. Department of Neurosurgery, Albany Medical Center, Albany, NY

\subsection{6/neurintsurg-2016-012589.18}

Introduction There has been an increasing use of flow diverting stents in the endovascular management of intracranial aneurysms which previously would have been considered difficult to treat. Currently there are two flow diverting stents available in the United States. The Pipeline Embolization Devi$\mathrm{ce}^{\mathrm{TM}}$ (Covidien) was approved by the FDA in 2011 and the Flow Redirection Endoluminal Stent (FRED ${ }^{\mathrm{TM}}$, MicroVention) is currently involved in a clinical trial. There have been no comparisons of the two devices to date.

Materials and methods Patients treated with any means of Flow Diversion over a 2 year period were identified. Records and images were reviewed retrospectively. Statistics were calculated in Excel.

Results Twenty one aneurysms were treated in 19 patients using the FRED stent and 42 aneurysms were treated in 36 patients using a Pipeline stent. The age of the FRED patients was significantly higher than the Pipeline patients. There was no difference between in other demographic characteristics (Table 1).

Conclusion We present a single institutional experience with two flow diverting stents. There was no significant difference in neurological morbidity or mortality. There was a trend 
towards a higher rate of complete occlusion with the FRED device.

Abstract 0-018 Table 1 The demographics of the two groups

\begin{tabular}{llll}
\hline & FRED & Pipeline & p-value \\
\hline Age $^{*}$ & $59.1 \pm 10.7$ & $49.1 \pm 13.8$ & 0.02 \\
Male & $4(21 \%)$ & $10(27.8 \%)$ & 0.7 \\
Previously ruptured & $1(5 \%)$ & $4(11 \%)$ & 0.65 \\
Retreatment(Previously coiled) & $4(21 \%)$ & $8(22 \%)$ & 1 \\
Coils Placed at the time of treatment & $1(5 \%)$ & $2(5 \%)$ & 1 \\
Multiple Devices & $3(16 \%)$ & $5(14 \%)$ & 1 \\
Fusiform & $2(11 \%)$ & $9(25 \%)$ & 0.3 \\
PRU at time of procedure & $122 \pm 63$ & $124 \pm 67$ & 0.9 \\
\hline
\end{tabular}

There was no significant difference between the complication rates for the two groups, including ipsilateral ischemic stroke, ipsilateral $\mathrm{ICH}$, aneurysm rupture and overall neurological morbidity and mortality.

\begin{tabular}{llll}
$\begin{array}{l}\text { Abstract 0-018 Table } 2 \\
\text { device }\end{array}$ & \multicolumn{3}{l}{ The complications associated with each } \\
\hline FRED & $\begin{array}{l}\text { ICA }>10 \mathrm{~mm} \\
(\mathbf{n}=10)\end{array}$ & $\begin{array}{l}\text { ICA }<10 \mathrm{~mm} \\
(\mathbf{n}=7)\end{array}$ & $\begin{array}{l}\text { All } \\
(\mathbf{n}=19)\end{array}$ \\
\hline $\begin{array}{l}\text { Ipsilateral Ischemic Stroke } \\
\text { Ipsilateral ICH }\end{array}$ & $1(10 \%)$ & $1(14 \%)$ & $2(10.5 \%)$ \\
Aneurysm Rupture & $0(0 \%)$ & $0(0 \%)$ & $0(0 \%)$ \\
Neurological Morbidity \& & $1(0 \%)$ & $0(0 \%)$ & $0(0 \%)$ \\
Mortality & $1(10 \%)$ & $1(14 \%)$ & $2(10.5 \%)$ \\
Pipeline & ICA $>10 \mathrm{~mm}$ & ICA $<10 \mathrm{~mm}$ & All \\
& $(\mathrm{n}=3)$ & $(\mathrm{n}=26)$ & $(\mathrm{n}=36)$ \\
Ipsilateral Ischemic Stroke & $0(0 \%)$ & $2(7.7 \%)$ & $3(8.3 \%)$ \\
Ipsilateral ICH & $0(0 \%)$ & $1(3.8 \%)$ & $1(2.7 \%)$ \\
Aneurysm Rupture & $0(0 \%)$ & $0(0 \%)$ & $0(0 \%)$ \\
Neurological Morbidity \& & $0(0 \%)$ & $3(11.5 \%)$ & $4(11.1 \%)$ \\
Mortality & & & \\
\hline
\end{tabular}

There was no significant difference in occlusion rate between the two groups. There was a trend towards a higher rate of complete occlusion in the FRED group but this was not statistically significant $(\mathrm{p}=0.17)$. When only saccular aneurysms were evaluated, the complete occlusion rate in the FRED group was $92.3 \%$ and $70 \%$ in the pipeline group $(\mathrm{p}=0.2$ ).

\begin{tabular}{lll} 
Abstract 0-018 Table 3 & Results of each device & \\
\hline FRED & Anterior & All \\
\hline 6 month angiographic complete occlusion & $11 / 13(84.6 \%)$ & $11 / 14(78.6 \%)$ \\
12 month angiographic complete occlusion & $7 / 8(87.5 \%)$ & $7 / 8(87.5 \%)$ \\
Any follow up imaging & $13 / 14(92.9 \%)$ & $13 / 15(86.7 \%)$ \\
Pipeline & Anterior & All \\
6 month angiographic complete occlusion & $7 / 9(77.8 \%)$ & $7 / 10(70 \%)$ \\
12 month angiographic complete occlusion & NA & NA \\
Any follow up imaging & $14 / 21(66.7 \%)$ & $17 / 26(65.4 \%)$ \\
\hline
\end{tabular}

Disclosures A. Paul: None. J. Dalfino: None. J. Yamamoto: None. A. Boulos: None.

\section{0-019 NOVEL METHOD FOR INTRACRANIAL DEPLOYMENT OF 6 AND 8 MM DIAMETER SELF EXPANDING COBALT CHROMIUM STENTS IN THE ARTERIAL AND VENOUS CIRCULATIONS}

C Martin, I Akhtar, J Halpin, W Holloway, N Akhtar. Marion Bloch Neuroscience Institute, Saint Luke's Hospital of Kansas City, Kansas City, MO

\subsection{6/neurintsurg-2016-012589.19}

Introduction/purpose Stents for the intracranial circulation are approved for stent assisted coiling of brain aneurysms, flow diversion of brain aneurysms and atherosclerotic occlusive disease. These stents are deliverable and approved for intracranial placement of cerebral arteries ranging in size from 2.5 to 4.5 $\mathrm{mm}$. The relatively small stents are unsuitable for skull based internal carotid artery for dissection and venous sinus deployment due to the large diameter of these vessels. Self-expandable cobalt chromium carotid stents are properly sized to these circulations but do not track the curvatures of the intracranial arterial and venous circulations. These self-expanding cobalt chromium carotid stents are available in 6 and $8 \mathrm{~mm}$ diameter sizes and, due to the braided design, these implants will track curves when partially deployed. We report a technique of partially deploying a cobalt chromium stent within a 072 intracranial support catheter which has already been positioned distal to the target. This allows the stent to be advanced around curves and unsheathed directly from the catheter thus allowing intracranial stenting of larger vessels.

Materials and methods This is a retrospective review of 11 cases in which cobalt chromium stents were placed in the intracranial circulation at our facility over a two year period. These stents were placed with an 072 intracranial support catheter positioned distal to the target. Three stents were placed in distal cervical ICA transitioning into the horizontal petrous ICA. Eight stents were placed in the venous circulation (1 straight sinus to transverse sinus, 5 transverse sinus, 1 transverse-sigmoid junction, 1 sigmoid-jugular junction). In two venous cases, the stents were used to support coil masses. Results All cases in which the cobalt chromium stents were deployed were successful. No neurological sequelae were observed post procedure.

Conclusion This technique allows for a wider range of stent sizes to access the tortuous intracranial circulation and provides an additional tool to the neurointerventional surgeon.

Disclosures C. Martin: None. I. Akhtar: None. J. Halpin: None. W. Holloway: None. N. Akhtar: None.

\section{0-020 WEB ${ }^{\circledR}$ ANEURYSM TREATMENT: PRELIMINARY RESULTS OF WEBCAST 2 STUDY}

L Pierot, A Molyneux, J Byrne. Radiology, Hôpital Maison-Blanche, Reims, France

\subsection{6/neurintsurg-2016-012589.20}

Purpose WEB Flow disruption is an innovative endovascular treatment for wide-neck bifurcation aneurysms. This treatment was already evaluated in several retrospective and prospective series, including 2 Good Clinical Practice (GCP) studies (WEBCAST and French Observatory) showing high safety and good efficacy. In WEBCAST, all patients were treated with the first generation of the device (WEB-DL) and WEBCAST2 was initiated to evaluate the second generation of the device (WEB-SL and WEB-SLS). 